\title{
EFFECT OF SALT MAGNESIUM CHLORIDE ON VAPOUR - LIQUID EQUILIBRIA OF BINARY AZEOTROPIC LIQUID MIXTURE: ETHYL ACETATE - ETHANOL
}

\author{
Shyam Tekade ${ }^{1}$, Sudesh Ayare ${ }^{2}$ \\ ${ }^{1}$ Assistant Professor, Chemical Engineering Department, Gharda Institute of Technology, Maharashtra, India \\ ${ }^{2}$ Assistant Professor, Chemical Engineering Department, Gharda Institute of Technology, Maharashtra, India
}

\begin{abstract}
Separation of azeotropic liquid mixtures using distillation is a challenging task for process engineers. A straight distillation is not useful for complete separation of mixture components for azeotropic liquid mixtures. The conventional process using liquid entrainer is energy intensive and involves running extra distillation column. The salt which dissolve in liquid mixture but has different solubility in components of liquid mixture can be used as the extracting agent like that of liquid entrainer. The added salt can hold the component in equilibrium liquid phase which has more solubility and hence can enrich the equilibrium vapour phase with the other component. Therefore the added salt has the capability to alter the relative volatility of liquid mixture. The Vapour - Liquid Equilibrium data indicates the relative amount of components in liquid and vapour phase and hence indicates extend up to which liquid mixture can be separated using distillation. In this paper we have studied the separation of one of the important azeotropic liquid mixture Ethyl Acetate -Ethanol in presence of salt Magnesium Chloride. The Vapour - Liquid Equilibrium (VLE) data of binary liquid mixture Ethyl Acetate (A)-Ethanol (B) without salt and with salt is obtained experimentally by using modified Othmer's still. The system found to forms the minimum boiling azeotrope at 0.550 mole fraction of ethyl acetate at 71.7 ${ }^{0} \mathrm{C}$ under the normal atmospheric pressure. The effect of addition of salt Magnesium Chloride $\left(\mathrm{MgCl}_{2}\right)$ has been studied on the vapour - liquid equilibria of ethyl acetate-ethanol. Two different compositions of salt $(20 \%$ and $25 \%$ by weight of ethanol in mixture) have been tried and corresponding shift in azeotropic point towards the higher composition of ethyl acetate than without salt is noted.
\end{abstract}

Keywords: VLE, binary, azeotrope, mole fraction, salt.

\section{INTRODUCTION}

The separation operation for separating the liquid mixtures into pure components constitutes one of the important aspects in chemical industries. Throughout the chemical industry, continuous demand for compositional purity of components with greater efficiency has imposed the everlasting research in designing efficient techniques of separation. The design of important equipment for separation like distillation column, absorption column, extraction column, humidifier, dehumidifier, etc. are depends on the equilibrium relationship of components involved in the system, between the different phases. Particularly, distillation is one of the important unit operations in chemical industries and distillation columns are to be designed with massive ranges than any other separating equipment. Industrially, the design of single distillation column can take the figures like 0.3 to $10 \mathrm{~m}$ in diameter and 3 to $75 \mathrm{~m}$ in height depending on the conditions of separation [1]. The design and operation of stage-wise or differential contacting equipment such as a distillation column require reliable vapor - liquid equilibrium data [2]. Azeotrope is the characteristics composition of liquid mixture for which the composition of equilibrium liquid and vapor phases are equal. Azeotrope is a result of intermolecular forces between the molecules of liquid at a given composition of mixture. Close boiling points of the pure components in the liquid mixture is one of the cause for formation of an azeotrope. Azeotropes occur infrequently for mixtures composed of components whose boiling points differ by more than $30^{\circ} \mathrm{C}$ [3]. As the liquid and vapor composition becomes same at azeotropic composition, it is not possible to separate the azeotropic liquid mixture beyond azeotropic compositions by using simple distillation. Industrially the extractive distillation is used to separate the azeotropic liquid mixtures in which the third liquid component also called as liquid entrainer added to binary mixture to affect the properties of system and facilitates separation. But, this added liquid transfers the phase and moves with the vapors during the operation and to recover (and recycle) it, an extra distillation column is needed [5]. One of the ways to overcome this difficulty is to use the solid salt as an extracting agent instead of liquid entrainer. The specific solid salts are found to alter the relative volatility of liquid mixtures to facilitate the separation of azeotropic liquid mixtures [5-7]. The solid salt cannot vaporized and transfers to vapor phase unlike of that liquid entrainer and this is advantageous over the conventional process as the additional distillation column is not required to recover the added entrainer. 
Kablukov was the first who observed the effect of salt on the partial pressure exerted over the aqueous ethanol. According to his observation salt more soluble in water than in ethanol increases the vapour pressure of alcohol and vice-versa. Changes in vapour pressure were proportional to the concentration of salt dissolved in the mixture. After this, much work has been done for the systems in which one of the components is water, with reference to the salt effect. Nearly same effects of salt on vapour liquid equilibrium have been noted. For non-aquous systems, Johnson, Ward, and Furter found that sodium propionate greatly increased the proportion of n-octane in the equilibrium vapour of boiling mixtures of n-octane-propionic acid, and shifted the azeotrope considerably. Walker et al also referred the use of salt effect to separate methyl alcohol from acetone. Suitable salts mentioned were sodium hydroxide, potassium carbonate, and sodium thiosulphate [7]. For acetone methanol system, L.Belcku studied the effect of calcium chloride, J. Proszt and G. Kollar also studied the effect of calcium-chloride and lithium-chloride. The isobaric vapour liquid equilibrium data of the same system by using six different salts $\mathrm{KI}, \mathrm{NaCl}, \mathrm{MgCl}_{2}, \mathrm{CaCl}_{2}, \mathrm{LiCl}$ and $\mathrm{CaBr}_{2}$ was discussed by S Ohe, K Yokoyama and S Nakamura [8]

The binary liquid mixture ethyl acetate- ethanol is observe to forms a minimum boiling azeotrope at 0.55 mole fraction of ethyl acetate at $71.7 \mathrm{~K}$ under the local atmospheric pressure $700 \mathrm{~mm} \mathrm{Hg}$. The system ethyl acetate - ethanol was studied and reported by Hashitani and Hirata with different salts, namely, zinc chloride, potassium acetate and calcium chloride [9]. Effect of salt on vapor liquid equilibrium of four binary system was studied by Feiyan $\mathrm{Gu}$ and Yujun Hou [10]. P Rath, S C Naik also studied the system ethyl acetate - ethanol by using the salts lithium chloride, lithium bromide and lithium iodide and reported the shift in azeotropic composition [11]. Recently, the same system has been studied by A. R. Balakrishnan and et al with different divalent chloride and nitrate salts at various salt concentrations and successfully correlated the data obtained [12]. The intension of this work is to observe the effect of salt Magnesium Chloride $\left(\mathrm{MgCl}_{2}\right)$ on vapor liquid equilibrium of binary azeotropic liquid mixture ethyl acetate- ethanol. Shift of azeotropic composition with respect to the saturation of liquid mixture with salt is studied by taking the two different composition of salt, one before saturation and another at saturation.

\section{EXPERIMENTAL}

\subsection{Apparatus}

The equilibrium still similar to modified Othmer still is used for determining vapor liquid equilibrium data [13]. The main parts of still are boiling pot, heater and condenser. Boiling pot is a flat bottom three-neck flask having capacity of $500 \mathrm{ml}$. This flask is completely lagged with thick layer of plaster of paris to have the insulation. The boiling pot is provided with opening to introduce the sample. The flask is also provided with two-thermometer pockets for inserting the thermometer. One thermometer is used to measure the temperature of liquid while other measures the temperature of vapor. Contents in the boiling pot are constantly stirred with the magnetic stirrer. The vapor line has arranged in such a way that after condensing the vapor, the condensate can be again recirculate to the boiling liquid through condensate chamber and three way valve. After reaching the equilibrium, condensate sample can be taken out for analysis through the three-way valve. Flat plate heater is used to mount the boiling pot.

\subsection{Experimental Procedure}

Initially the boiling pot is thoroughly washed with water and then with acetone. A liquid mixture sample of about $200 \mathrm{ml}$ of known composition was prepared in a measuring cylinder. For each run, such mixtures were prepared by progressively increasing one of the components while decreasing the other. The heater, magnetic stirrer, and condenser water was turn on. The temperature of liquid starts rising slowly and finally boiling takes place after half an hour. The steady state was attained in half an hour. During the run, vapors are totally condensed in condenser and returned to boiling liquid through the three-way valve. Though the equilibrium attained in initial 30 minutes the boiling and circulation of condensate was continued about one and half hour. After one and half hour, the condensate and liquid from the still was taken for analysis. Here, for analyzing the liquid which is in equilibrium with vapor, the same procedure as adopted by Shuzo Ohe and et .al. [14] was used. Only the equilibrium vapor condensate samples were analyzed. The concentrations of ethyl acetate and ethanol in equilibrium liquid phase were calculated by mass balance, using the concentration of ethyl acetate and ethanol in original charge and analyzed concentration of ethyl acetate and ethanol in equilibrium vapor samples. Specific gravity measurements using gravity bottle and refractive index measurement by using digital refractometer is used to analyze the equilibrium liquid and vapor compositions. Two different concentration of salt Magnesium Chloride are used to study the effect of salt on vapor liquid equilibrium of ethyl acetate ethanol system. Vapor liquid equilibrium data of ethyl acetate - ethanol is obtained (i) without salt (ii) with $20 \% \mathrm{MgCl}_{2}$ by weight of ethanol (iii) with $25 \% \mathrm{MgCl}_{2}$ by weight of ethanol.

\subsection{Materials}

The used liquid chemicals ethyl acetate and ethanol chemicals are supplied by S. D. Finechem Ltd, Mumbai, and are of analytical reagents grade. The physical properties of chemicals used are compared with the literature values and given in the table 1 .

Table -1: Physical properties of chemicals used

\begin{tabular}{|l|l|l|l|l|}
\hline \multirow{2}{*}{ Component } & \multicolumn{2}{|l|}{ Boiling Point, ${ }^{0} \mathrm{C}$} & \multicolumn{2}{l|}{ Specific gravity } \\
\cline { 2 - 5 } & $\begin{array}{l}\text { Reference } \\
{[15]}\end{array}$ & $\begin{array}{l}\text { Present } \\
\text { Study }\end{array}$ & $\begin{array}{l}\text { Reference } \\
{[15]}\end{array}$ & $\begin{array}{l}\text { Present } \\
\text { Study }\end{array}$ \\
\hline $\begin{array}{l}\text { Ethyl } \\
\text { acetate }\end{array}$ & 76.10 & 75.20 & 0.901 & 0.897 \\
\hline Ethanol & 78.40 & 78.29 & 0.787 & 0.788 \\
\hline
\end{tabular}


The solid salt is supplied by Fisher Scientific Ltd, and of analytical reagent grade. Prior to use it in experiment to remove the moisture content, salt is dried for six hours in hot air oven at $105^{\circ} \mathrm{C}$ and stored in a desiccator.

\section{CONCLUSION}

The VLE data of ethyl acetate-ethanol without salt is given in table 2. The VLE diagram of ethyl acetate -ethanol in absence of salt is shown in chart 1 . The minimum boiling azeotrope is observed at 0.55 mole fraction of ethyl acetate. The relative solubility of salt $\mathrm{MgCl}_{2}$ in ethanol and ethyl acetate is then checked by general method. It is observe that the salt $\mathrm{MgCl}_{2}$ dissolves more in ethanol as compare to ethyl acetate. After trying some initial concentrations of salt, it is decided to take the two compositions i.e. $20 \%$ and $25 \%$ of the weight of ethanol in solution.

Table -2: VLE Data for ethyl acetate - ethanol without salt

\begin{tabular}{|l|l|l|l|l|l|}
\hline $\mathrm{x}$ & $\mathrm{y}$ & $\mathrm{T}^{0} \mathrm{C}$ & $\gamma_{1}$ & $\gamma_{2}$ & $\alpha$ \\
\hline 1 & 1 & 75.2 & -- & -- & \\
\hline 0.875 & 0.79 & 72.5 & 0.9697 & 1.9581 & 0.9028 \\
\hline 0.755 & 0.65 & 71.9 & 0.9435 & 1.7067 & 0.8609 \\
\hline 0.58 & 0.56 & 71.7 & 1.0653 & 1.2620 & 0.9655 \\
\hline 0.425 & 0.495 & 71.8 & 1.2807 & 1.0536 & 1.1647 \\
\hline 0.315 & 0.425 & 71.9 & 1.4786 & 1.0028 & 1.3492 \\
\hline 0.25 & 0.375 & 72.6 & 1.6057 & 0.9673 & 1.5000 \\
\hline 0.165 & 0.305 & 73.2 & 1.9395 & 0.9426 & 1.8484 \\
\hline 0.085 & 0.215 & 74.8 & 2.5167 & 0.9102 & 2.5294 \\
\hline 0.05 & 0.125 & 76 & 2.3913 & 0.9310 & 2.5000 \\
\hline 0 & 0 & 78.2 & -- & -- & -- \\
\hline
\end{tabular}

For $20 \% \mathrm{MgCl}_{2}$ the VLE data is given in table 3 and VLE diagram is as shown in chart 2. For $25 \% \mathrm{MgCl}_{2}$ the VLE data is given in table 3 and VLE diagram is as shown in chart 2 .

It is observed that $20 \%$ concentration of salt shifts the azeotropic point from 0.55 to 0.625 mole fraction of ethyl acetate. This shift in azeotrope represents the ability of salt $\mathrm{MgCl}_{2}$ to salt out ethyl acetate. As the salt is more soluble in ethanol, ethanol has the greater affinity than ethyl acetate for $\mathrm{MgCl}_{2}$ which results in increase of concentrations of ethyl acetate (salting out) in vapor. For initial low concentrations of ethyl acetate $\mathrm{MgCl}_{2}$ gets completely dissolve in solution. As the concentration of ethyl acetate increases in solution, solubility of $\mathrm{MgCl}_{2}$ decreases and some $\mathrm{MgCl}_{2}$ starts appearing in solution. So, for some initial low concentrations of ethyl acetate, the solution remains unsaturated with salt. As the concentrations of ethyl acetate in solution increases the solution progresses towards the saturation.

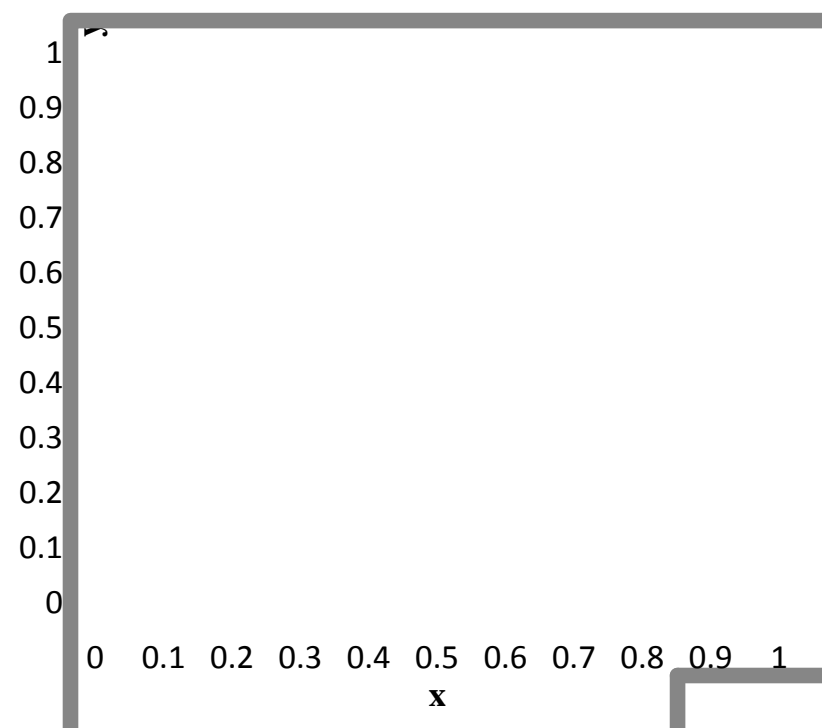

Chart -1: VLE Diagram of Ethyl Acetate- Ethanol without Salt

Table -3: VLE Data for ethyl acetate - ethanol $20 \% \mathrm{MgCl}_{2}$

\begin{tabular}{|l|l|l|l|l|l|}
\hline & & & & & \\
& & & & & \\
& & & \\
\hline 1.000 & 1.000 & 75.2 & -- & -- & -- \\
\hline 0.891 & 0.725 & 72 & 0.8887 & 3.0018 & 0.8136 \\
\hline 0.706 & 0.69 & 71.8 & 1.0747 & 1.2649 & 0.9773 \\
\hline 0.633 & 0.619 & 71.7 & 1.0789 & 1.2506 & 0.9778 \\
\hline 0.508 & 0.605 & 71.9 & 1.3052 & 0.9592 & 1.1909 \\
\hline 0.447 & 0.6 & 72.04 & 1.4641 & 0.8592 & 1.3422 \\
\hline 0.327 & 0.53 & 72.7 & 1.7292 & 0.8073 & 1.6207 \\
\hline 0.25 & 0.508 & 73.4 & 2.1179 & 0.7368 & 2.032 \\
\hline 0.155 & 0.39 & 74.2 & 2.5537 & 0.7848 & 2.5161 \\
\hline 0.047 & 0.245 & 75.1 & 5.1356 & 0.8304 & 5.2127 \\
\hline 0.000 & 0.000 & 78.2 & -- & -- & -- \\
\hline
\end{tabular}




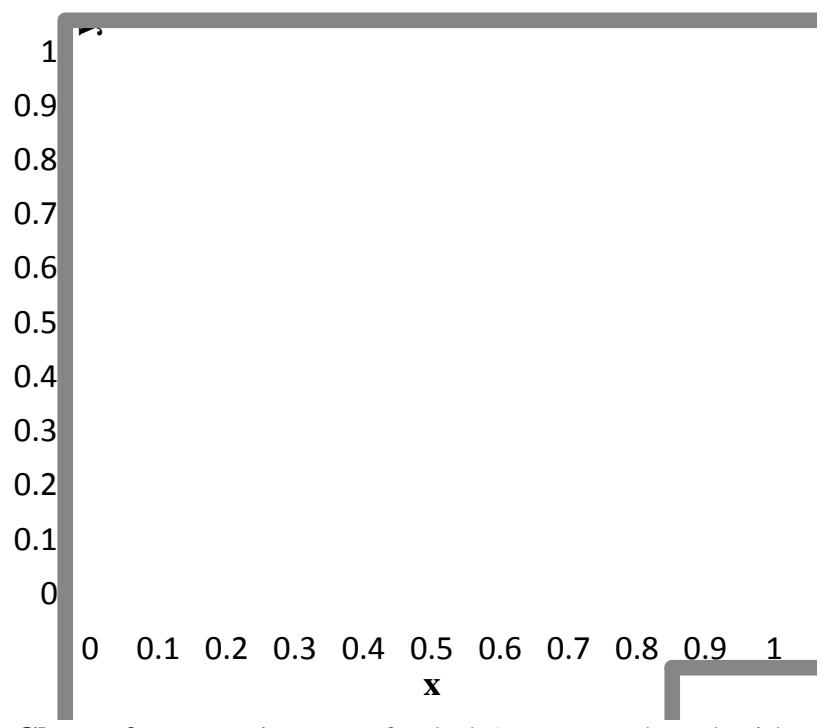

Chart -2: VLE Diagram of Ethyl Acetate- Ethanol with $20 \% \mathrm{MgCl}_{2}$

Table -4: VLE Data for ethyl acetate - ethanol with 25\% salt

\begin{tabular}{|l|l|l|l|l|l|}
\hline $\mathrm{x}$ & $\mathrm{y}$ & $\mathrm{T}^{0} \mathrm{C}$ & $\gamma_{1}$ & $\gamma_{2}$ & $\alpha$ \\
\hline 1 & 1 & 75.2 & -- & -- & -- \\
\hline 0.96 & 0.82 & 73.8 & 0.8785 & 4.9727 & 0.8541 \\
\hline 0.71 & 0.66 & 71.8 & 1.0222 & 1.4065 & 0.9295 \\
\hline 0.615 & 0.624 & 70.9 & 1.1502 & 1.2162 & 1.0146 \\
\hline 0.51 & 0.62 & 71.0 & 1.3734 & 0.9617 & 1.2156 \\
\hline 0.472 & 0.609 & 71.1 & 1.4527 & 0.9145 & 1.2902 \\
\hline 0.392 & 0.587 & 71.2 & 1.6803 & 0.8354 & 1.4974 \\
\hline 0.265 & 0.47 & 71.2 & 1.9902 & 0.8868 & 1.7735 \\
\hline 0.18 & 0.402 & 72.5 & 2.3988 & 0.8499 & 2.2333 \\
\hline 0.07 & 0.255 & 74.0 & 3.7218 & 0.8780 & 3.6428 \\
\hline 0 & 0 & 78.2 & -- & -- & -- \\
\hline
\end{tabular}

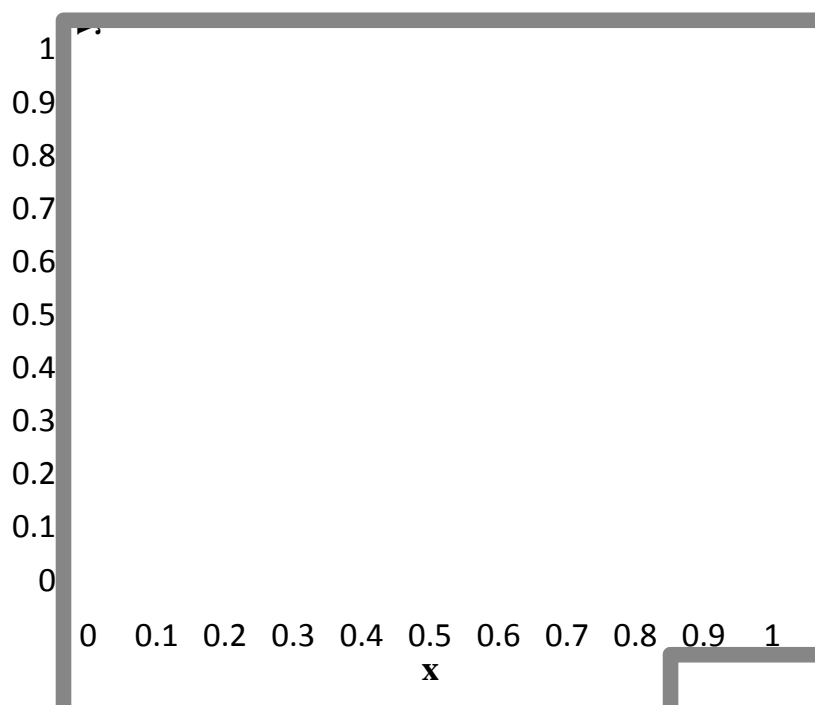

Chart -3: VLE Diagram of Ethyl Acetate- Ethanol with $25 \% \mathrm{MgCl}_{2}$

The $25 \%$ concentration of salt shifts the azeotropic point to 0.625 mole fraction of ethyl acetate. At the salt concentration $25 \%$ of the total weight of ethanol in solution, the solution always saturated with salt. As the saturation of solution occurs at this composition of salt, the possibility of shifting the azeotrope further (greater than 0.660 mole fraction of ethyl acetate) with even greater concentration of salt Magnesium Chloride is very rare. Hence, it is concluded that the salt Magnesium Chloride can shift the azeotrope of binary liquid mixture ethyl acetate-ethanol maximum up to 0.660 mole fraction of ethyl acetate i.e. Magnesium Chloride is not capable to remove the azeotrope of ethyl acetate-ethanol completely.

Activity coefficient $(\gamma)$ represents the deviation from the ideal behavior and can be calculated as:

$$
\gamma=\frac{y_{i} \times P}{x_{i} p_{i}^{s}}
$$

Where, $y_{i}$ is the mole fraction of component $i$ in vapor phase, $\mathrm{P}$ is the total pressure, $\mathrm{x}_{\mathrm{i}}$ mole fraction of component $\mathrm{i}$ in liquid phase and $\mathrm{p}_{\mathrm{i}}^{\mathrm{S}}$ is the saturation vapor pressure of the component i. Vapor pressure of pure components are calculated from Antoine equation. Constant for Antoine equation is taken from one of the reference. ${ }^{16}$ Activity coefficients $(\alpha)$ are listed in respective tables along with the VLE data. The relative volatility can be calculated by:

$$
\alpha=\frac{y_{1} / x_{1}}{y_{2} / x_{2}}
$$

Relative volatility values for respective composition of salt represented in tables along with VLE data. It is observe that, the relative volatility of the ethyl acetate -ethanol mixture enhances due to the addition of salt. After attaining the saturation there will be no further change in relative volatility. 


\section{REFERENCES}

[1]. Coulson J. M., Richardson J. F., Chemical Engineering: Volume 2, Butterworth-Heinemann Imprint (2002).

[2]. Paul R. N., Study of Liquid-Vapour Equilibrium in Improved Equilibrium Still, J. Chem. and Engg. Data, 21 (2), 165 (1976)

[3]. Perry R. H, Don Green, Perry's Chemical Engineering Handbook eight ed. McGraw Hill Pub. (2012)

[4]. Shreve R.N., George T. A., Shreve's Chemical Process Industries. McGraw-Hill Pub. (1984)

[5]. Tan T.C., Gan S.H., Vapour-Liquid Equilibrium of Water/Ethanol/1-Butanol/Salt Prediction and Experimental Verification, Chem. Engg. Res. Design, 83 (A12), 1361 (2005)

[6]. Chou T.J., Tanioka A., Predicting The Effect of Dissolved Salt on The Vapour-Liquid Equilibria For Alcohol-Water-Salt Systems, Trans I ChemE, 77 (A), 329 (1999)

[7]. Furter W.F., Derek J., Effect of a Dissolved Salt on Vapor-Liquid Equilibrium with Liquid Composition Held Constant, Ind. Eng. Chem. Fundam., 13(3), 238 (1974)

[8]. Furter W.F., Cook R.A., Salt Effect In Distillation: A Literature Review, Int. J. Heat Mass Transfer, 10, 23 (1967)

[9]. Motoyoshi H., Hirata M., Salt Effect in Vapor-Liquid Equilibrium Acetic Ester-Alcohol with Potassium Acetate and Zinc Chloride, J. of Chem. Engg. of Japan, 2 (2), 149 (1969)

[10]. Feiyan G., Yujun H., Salt Effects on the Isobaric Vapor-Liquid Equilibrium for Four Binary Systems, J. Chem. Eng. Data, 45, 467 (2000)

[11]. Rath P.,Naik S.C.,Prediction of Salt Effect in Vapourliquid Equilibria of System Ethyl Acetate-ethanol at Atmospheric Pressure, IE (I) J.CH, 84, (2004).

[12]. Dhanalakshmi J, Sai P.S.T., Balakrishnan A.R., Effect of Inorganic Salts on the Isobaric Vapor-Liquid Equilibrium of the Ethyl Acetate-Ethanol, J. Chem. Eng. Data, 58 (3), 560 (2013)

[13]. Morrison J.F., Baker J.C., Meredith H.C., Newman K.E., Waiter T.D.,Massle J.D., Perry R.L., Cummings P., Experimental Measurement of Vapor-Liquid Equilibrium in Alcohol/Water/Salt Systems, J. Chem. Eng. Data, 35, 395, (1900)

[14]. Ohe S., Yokoyama K., Nakamura S., Vapor-Liquid Equilibrium Data For The System Acetone-Methanol Saturated With Salts, J. chem Engg. Japan, 2, 1, (1969)

[15]. Speight J., Lange's Handbook of Chemistry 11th Ed., McGraw Hill Pub., (1973)

[16]. Himmelblau D., Riggs J. B. Basic Principles and Calculations in Chemical Engineering, PHI Pub.(2003) 